\title{
A $\boldsymbol{\mu}$-Pyrazolyl Terpyridineplatinum(II) Dimer
}

\author{
By James A. BAILEY* AND HARRY B. Gray \\ Division of Chemistry and Chemical Engineering $\dagger$ and The Beckman Institute, Mail Code 139-74, \\ California Institute of Technology, Pasadena, California 91125, USA
}

(Received 16 September 1991; accepted 14 January 1992)

\begin{abstract}
Pyrazolyl- $\left.N: N^{\prime}\right)$-bis $\left[\left(2,2^{\prime}, 2^{\prime \prime}\right.\right.$-terpyridine)platinum(II)] perchlorate acetonitrile solvate, $\left[\mathrm{Pt}_{2}\left(\mathrm{C}_{15} \mathrm{H}_{11} \mathrm{~N}_{3}\right)_{2}\left(\mathrm{C}_{3} \mathrm{H}_{3} \mathrm{~N}_{2}\right)\right]\left(\mathrm{ClO}_{4}\right)_{3} \cdot \mathrm{CH}_{3} \mathrm{CN}, \quad M_{r}=$ 1263.18, orthorhombic, Pnma, $a=18.172$ (2), $b=$ 17.950 (3), $c=12.086$ (4) $\AA, V=3942.3(15) \AA^{3}, Z=$ $4, D_{x}=2.128 \mathrm{~g} \mathrm{~cm}^{-3}, \lambda($ Mo $K \alpha)=0.71073 \AA, \mu=$ $74.41 \mathrm{~cm}^{-1}, \quad F(000)=2416$, room temperature $(297 \mathrm{~K}), R=0.0337$ for 2510 reflections with $F_{o}^{2}>$ $3 \sigma\left(F_{o}^{2}\right)$. A single pyrazolyl unit bridges two $\mathrm{Pt}$ centres. The square-planar coordination around each $\mathrm{Pt}$ atom is completed by a tridentate terpyridine ligand. The Pt $\cdots \mathrm{Pt}$ separation is 3.432 (1) $\AA$ with an inclination of $47.7(12)^{\circ}$ between the two Pt square planes.
\end{abstract}

Introduction. We are currently investigating the properties of binuclear terpyridineplatinum(II) complexes. The compound $(\mu-\mathrm{pz})$ bis[(tpy)Pt] $\left(\mathrm{ClO}_{4}\right)_{3}(\mathrm{pz}$ $=$ pyrazolyl, tpy $=$ terpyridine) is one of a series of binuclear complexes that have been synthesized in which the interplatinum distance is varied. The effect of the Pt-atom separation on absorption and emission spectra can be readily studied with these complexes.

Experimental. This material was synthesized by heating an aqueous solution of $[\mathrm{Pt}(\mathrm{tpy}) \mathrm{Cl}] \mathrm{Cl}$ and pyrazole ( $1 / 2$ equiv.) ( $\mathrm{pH}$ adjusted to $\mathrm{ca} 9$ with $1 M \mathrm{NaOH}$ ) to $348 \mathrm{~K}$ for $5 \mathrm{~d}$. The orange-red solution slowly changed to pale orange. Cooling and addition of $\mathrm{NaClO}_{4}$ precipitated the product as a perchlorate salt. Crystals for $\mathrm{X}$-ray analysis were grown by slow evaporation of an acetonitrile solution. A pale orange tabular block crystal $0.22 \times 0.42$ $\times 0.60 \mathrm{~mm}$ was used for data collection on a CAD-4 diffractometer, with $\omega$-scan technique. 25 reflections with $34<2 \theta<41^{\circ}$ were used for determination of the cell dimensions. An empiricial absorption correction based on $\psi$ scans of six reflections, with relative transmission from 0.508 to 1.388 , was applied. $(\sin \theta / \lambda)_{\max }=0.54 \AA^{-1} ; h$ from -14 to $14, k$ from -21 to $21, l$ from 0 to 17 . Three standard reflections

\footnotetext{
* To whom correspondence should be addressed. $\uparrow$ Contribution No. 8503.
}

0108-2701/92/081420-03\$06.00
$(153,411, \overline{3} 42)$ showed decay of $0.6 \%$ in $148 \mathrm{~h}$ of data collection; data were corrected for this decay. 14841 reflections were measured, of which 3588 were independent. Goodness of fit for merging was 1.35 $\left(R_{\text {merge }}\right.$ for 378 reflections with exactly two observations, 0.0175). All reflections were used in solution and refinement of the structure. Coordinates of the $\mathrm{Pt}$ atom were found from a Patterson map, remaining heavy atoms were found by successive structure factor - Fourier calculations, including an acetonitrile molecule of crystallization and two perchlorate ions. $F_{o}^{2}$ magnitudes were used in fullmatrix least-squares refinement, which minimized $\sum w\left(F_{o}^{2}-F_{c}^{2}\right)^{2} . \mathrm{H}$ atoms were positioned by calculation $(\mathrm{C}-\mathrm{H}=0.95 \AA)$ and included as constant contributors to the structure factors. $\mathrm{H}$ atoms on the acetonitrile were calculated for two possible positions based on the symmetry of the mirror plane which contains the $\mathrm{CH}_{3} \mathrm{CN}$ molecule; difference and Fourier maps did not favor either conformation so both positions were included, each contributor being set at half occupancy. $\mathrm{H}$-atom parameters were not refined but recalculated once, near the conclusion of the refinement. Positional and anisotropic temperature parameters for all non- $\mathrm{H}$ atoms (except $\mathrm{O}$ in disordered $\mathrm{ClO}_{4}^{-}$, isotropic), a scale factor and secondary-extinction parameter were refined. At convergence, difference maps suggested that the second $\mathrm{ClO}_{4}^{-}$ion which occupies a special position on a mirror plane might be modeled more appropriately as the superposition of two ions each with half occupancy and sharing both the $\mathrm{Cl} 2$ and $\mathrm{O} 6$ positions. Although this model accounts for most of the electron density in the area and the average $\mathrm{Cl}-\mathrm{O}$ bond length is $1.43 \AA$, the actual bond lengths and angles deviate substantially from a tetrahedral $\mathrm{ClO}_{4}^{-}$ geometry; however, other disorder models did not improve on this result, so refinement was terminated at this stage.

$R=0.0514$ for 3346 reffections with $F_{o}^{2}>0, w R$ (on $\left.F^{2}\right)=0.0050$ and goodness of fit $=2.01$ for 3588 reflections and 293 parameters. Weights were taken as $1 / \sigma^{2}\left(F_{o}^{2}\right)$; variances $\left[\sigma^{2}\left(F_{o}^{2}\right)\right]$ were derived from counting statistics plus an additional term, $\left(0.014 I^{2}\right.$; variances of the merged data by propagation of error

(C) 1992 International Union of Crystallography 
Table 1. Final refined atomic coordinates $\left(\times 10^{4}\right)$ and equivalent isotropic $\left(\AA^{2} \times 10^{4}\right) /$ isotropic $\left(\AA^{2}\right)$ temperature factors for $\mu$-pyrazolyl terpyridineplatinum dimer

$$
U_{\mathrm{eq}}=(1 / 3) \sum_{i} \sum_{j} U_{i j} a_{i}^{*} a_{j}^{*} \mathbf{a}_{i} \cdot \mathbf{a}_{j} .
$$

\begin{tabular}{|c|c|c|c|c|}
\hline & $x$ & $y$ & $z$ & $U_{\mathrm{eq}} / B^{\dagger}$ \\
\hline Pt & $769(.1)$ & $1544(.1)$ & $773(.2)$ & $436(1)$ \\
\hline N1 & 1651 (3) & $2128(3)$ & $1306(4)$ & 519 (14) \\
\hline N2 & $221(3)$ & $1425(3)$ & $2227(5)$ & 531 (16) \\
\hline N3 & $-97(3)$ & $1032(3)$ & $230(5)$ & 482 (15) \\
\hline N4 & $1067(3)$ & $1445(3)$ & $-829(5)$ & $550(16)$ \\
\hline $\mathrm{Cl}$ & 2318 (4) & 1911 (4) & $1652(7)$ & $742(26)$ \\
\hline $\mathrm{C} 2$ & 2745 (5) & 2500 & $1840(10)$ & $676(33)$ \\
\hline $\mathrm{C} 3$ & 443 (4) & $1620(5)$ & $3238(6)$ & $619(20)$ \\
\hline $\mathrm{C} 4$ & $10(5)$ & $1515(5)$ & $4129(7)$ & $777(25)$ \\
\hline C5 & $-681(5)$ & $1190(5)$ & $4040(7)$ & $756(26)$ \\
\hline C6 & $-899(4)$ & $966(5)$ & $3011(7)$ & $670(23)$ \\
\hline C7 & $-447(4)$ & $1081(4)$ & $2096(6)$ & 519 (19) \\
\hline $\mathrm{C} 8$ & $-630(3)$ & $874(4)$ & $965(6)$ & 501 (21) \\
\hline $\mathrm{C} 9$ & $-1271(4)$ & $542(4)$ & $570(8)$ & 653 (24) \\
\hline $\mathrm{C} 10$ & $-1347(4)$ & $426(5)$ & $-521(8)$ & $710(24)$ \\
\hline $\mathrm{Cll}$ & $-797(5)$ & $604(5)$ & $-1273(7)$ & $746(25)$ \\
\hline $\mathrm{Cl} 2$ & $-153(4)$ & $920(4)$ & $-872(6)$ & $518(20)$ \\
\hline $\mathrm{Cl} 3$ & $511(5)$ & $1127(4)$ & $-1465(7)$ & $596(23)$ \\
\hline $\mathrm{Cl} 4$ & $631(5)$ & $993(5)$ & $-2565(8)$ & 804 (27) \\
\hline $\mathrm{C} 15$ & $1319(7)$ & $1148(6)$ & $-3024(8)$ & $899(31)$ \\
\hline $\mathrm{Cl} 6$ & $1856(5)$ & $1429(5)$ & $-2375(9)$ & 878 (29) \\
\hline $\mathrm{C} 17$ & $1725(4)$ & $1580(4)$ & $-1281(7)$ & $667(21)$ \\
\hline $\mathrm{Cll}$ & $1776(1)$ & $169(1)$ & $3969(2)$ & $637(5)$ \\
\hline Ol & $1012(3)$ & $12(3)$ & $3858(5)$ & 844 (17) \\
\hline $\mathrm{O} 2$ & $1883(3)$ & $920(3)$ & $4340(5)$ & $933(19)$ \\
\hline $\mathrm{O} 3$ & 2096 (3) & $-323(4)$ & $4753(5)$ & $1018(20)$ \\
\hline $\mathrm{O} 4$ & $2142(3)$ & $103(4)$ & 2928 (5) & 985 (19) \\
\hline $\mathrm{Cl} 2$ & $3943(2)$ & 2500 & 8099 (3) & $971(12)$ \\
\hline O5 & 4294 (11) & 2500 & $6855(18)$ & $9.7(6)^{\dagger}$ \\
\hline O6 & $4620(10)$ & 2500 & 8521 (14) & $17.9(6) \dagger$ \\
\hline O7 & $3500(12)$ & $2097(12)$ & $7490(17)$ & $16.4(7) \dagger$ \\
\hline $\mathrm{O} 8$ & $3366(14)$ & 2500 & $9120(19)$ & $11.9(7) \dagger$ \\
\hline $\mathrm{O} 9$ & 3944 (9) & 1774 (11) & $8288(14)$ & $10.8(5) \dagger$ \\
\hline N5 & 3957 (8) & 2500 & 4114 (13) & $1237(50)$ \\
\hline $\mathrm{C} 18$ & $2712(9)$ & 2500 & $5083(12)$ & $1037(49)$ \\
\hline $\mathrm{C} 19$ & $3412(9)$ & 2500 & $4573(12)$ & $837(43)$ \\
\hline
\end{tabular}

$\uparrow$ Isotropic displacement parameter.

Table 2. Selected distances $(\AA)$ and angles $\left(^{\circ}\right)$ for $\mu$-pyrazolyl terpyridineplatinum dimer

$\begin{array}{ll}\mathrm{Pt}-\mathrm{N} 1 & 2.020(5) \\ \mathrm{Pt}-\mathrm{N} 3 & 1.938(5) \\ \mathrm{N} 1-\mathrm{C} 1 & 1.340(9) \\ \mathrm{N} 2-\mathrm{C} 3 & 1.334(10) \\ \mathrm{N} 3-\mathrm{C} 8 & 1.345(9) \\ \mathrm{N} 4-\mathrm{C} 13 & 1.391(10) \\ \mathrm{C} 1-\mathrm{C} 2 & 1.331(13) \\ \mathrm{C} 4-\mathrm{C} 5 & 1.388(13) \\ \mathrm{C} 6-\mathrm{C} 7 & 1.393(11) \\ \mathrm{C} 8-\mathrm{C} 9 & 1.392(11) \\ \mathrm{C} 10-\mathrm{C} 11 & 1.389(12) \\ \mathrm{C} 12-\mathrm{C} 13 & 1.451(11) \\ \mathrm{C} 14-\mathrm{C} 15 & 1.395(14) \\ \mathrm{C} 16-\mathrm{C} 17 & 1.371(13)\end{array}$

$\begin{array}{lr}\mathrm{N} 2-\mathrm{Pt}-\mathrm{N} 1 & 99.7(2) \\ \mathrm{N} 4-\mathrm{Pt}-\mathrm{N} 1 & 98.0(2) \\ \mathrm{N} 4-\mathrm{P} 1-\mathrm{N} 2 & 162.2(2) \\ \mathrm{Cl}-\mathrm{N} 1-\mathrm{Pt} & 131.8(5) \\ \mathrm{N} 1-\mathrm{N} 1-\mathrm{Cl} & 106.9(5) \\ \mathrm{C} 7-\mathrm{N} 2-\mathrm{Pt} & 112.5(4) \\ \mathrm{C} 8-\mathrm{N} 3-\mathrm{Pt} & 117.5(4) \\ \mathrm{C} 12-\mathrm{N} 3-\mathrm{C} 8 & 124.5(6) \\ \mathrm{C} 17-\mathrm{N} 4-\mathrm{Pt} & 128.0(5) \\ \mathrm{C} 2-\mathrm{Cl}-\mathrm{N} 1 & 110.5(8) \\ \mathrm{C} 4-\mathrm{C} 3-\mathrm{N} 2 & 121.3(7) \\ \mathrm{C} 6-\mathrm{C} 5-\mathrm{C} 4 & 117.2(8) \\ \mathrm{C} 6-\mathrm{C} 7-\mathrm{N} 2 & 119.9(6) \\ \mathrm{C} 8-\mathrm{C} 7-\mathrm{C} 6 & 124.9(7) \\ \mathrm{C} 9-\mathrm{C} 8-\mathrm{N} 3 & 117.8(6) \\ \mathrm{C} 10-\mathrm{C} 9-\mathrm{C} 8 & 119.3(7) \\ \mathrm{C} 12-\mathrm{C} 11-\mathrm{C} 10 & 118.2(8) \\ \mathrm{C} 13-\mathrm{C} 12-\mathrm{N} 3 & 112.8(6) \\ \mathrm{C} 12-\mathrm{C} 13-\mathrm{N} 4 & 115.8(7) \\ \mathrm{C} 14-\mathrm{C} 13-\mathrm{C} 12 & 124.6(7) \\ \mathrm{C} 16-\mathrm{C} 15-\mathrm{C} 14 & 119.4(9) \\ \mathrm{C} 16-\mathrm{C} 17-\mathrm{N} 4 & 120.8(8)\end{array}$

$\begin{array}{ll}\mathrm{Pt}-\mathrm{N} 2 & 2.031(6) \\ \mathrm{Pt}-\mathrm{N} 4 & 2.018(6) \\ \mathrm{N} 1-\mathrm{N} 1 & 1.335(7) \\ \mathrm{N} 2-\mathrm{C} 7 & 1.370(9) \\ \mathrm{N} 3-\mathrm{C} 12 & 1.350(9) \\ \mathrm{N} 4-\mathrm{C} 17 & 1.337(10) \\ \mathrm{C} 3-\mathrm{C} 4 & 1.347(12) \\ \mathrm{C} 5-\mathrm{C} 6 & 1.366(12) \\ \mathrm{C} 7-\mathrm{C} 8 & 1.456(10) \\ \mathrm{C} 9-\mathrm{C} 10 & 1.342(12) \\ \mathrm{C} 11-\mathrm{C} 12 & 1.388(11) \\ \mathrm{C} 13-\mathrm{C} 14 & 1.370(12) \\ \mathrm{C} 15-\mathrm{C} 16 & 1.349(14)\end{array}$

$\mathrm{N} 3-\mathrm{Pt}-\mathrm{N} 1 \quad 177.0(2)$

$\mathrm{N} 3-\mathrm{Pt}-\mathrm{N} 2$

$\mathrm{N} 4-\mathrm{Pt}-\mathrm{N} 3 \quad 81.4(2)$

$\mathrm{NI}-\mathrm{NI}-\mathrm{Pt} \quad 121.3(4)$

$\mathrm{C} 3-\mathrm{N} 2-\mathrm{Pt} \quad 128.1(5)$

C7-N2-C3 $\quad 119.4(6)$

$\mathrm{C} 12-\mathrm{N} 3-\mathrm{Pt} \quad 117.7(4)$

$\mathrm{C} 13-\mathrm{N} 4-\mathrm{Pt} \quad 111.8(5)$

$\mathrm{C} 17-\mathrm{N} 4-\mathrm{C} 13$

$\mathrm{Cl}-\mathrm{C} 2-\mathrm{Cl} \quad 105.1(9)$

$\mathrm{C} 5-\mathrm{C} 4-\mathrm{C} 3 \quad 121.7(8)$

$\mathrm{C} 7-\mathrm{C} 6-\mathrm{C} 5 \quad 120.5(7)$

$\mathrm{C} 8-\mathrm{C} 7-\mathrm{N} 2 \quad 115.2(6)$

$\mathrm{C} 7-\mathrm{C} 8-\mathrm{N} 3 \quad 113.7(6)$

$\mathrm{C} 9-\mathrm{C} 8-\mathrm{C} 7 \quad 128.5(7)$

$\mathrm{C} 11-\mathrm{C} 10-\mathrm{C} 9 \quad 122.2(8)$

$\mathrm{C} 11-\mathrm{Cl} 2-\mathrm{N} 3 \quad 117.9(7)$

C13-C12-C11 $\quad 129.3(7)$

$\mathrm{C} 14-\mathrm{Cl}-\mathrm{N} 4 \quad 119.5(7)$

$\mathrm{C} 15-\mathrm{C} 14-\mathrm{C} 13 \quad 119.6(8)$

$\mathrm{C} 17-\mathrm{C} 16-\mathrm{C} 15 \quad 120.6(9)$ plus another additional term, $(0.014 \bar{I})^{2}$. In the final least-squares cycles $(\Delta / \sigma)_{\max }=0.04 ;-0.91 \leq \Delta \rho \leq$ $1.57 \mathrm{e}^{-3}$ in the final difference Fourier map, with all large peaks within $1.5 \AA$ of the $\mathrm{Pt}$ atom. Atomic scattering factores and values for $f^{\prime}$ were taken from Cromer \& Waber (1974) and Cromer (1974). Programs used were those of the CRYM crystallographic computing system (Duchamp, 1964) and ORTEPII (Johnson, 1976). Final atomic coordinates and $U_{\text {eq }}$ are listed in Table 1 with selected distances and angles given in Table 2.*

Discussion. Fig. 1 shows the cation viewed along the intermetal axis with atom labels; Fig. 2 is a stereoscopic view of the entire $3+$ cation. The geometry around the $\mathrm{Pt}$ atoms is nearly square planar, distorted only by the restricted bite angle of the planar

* Lists of assigned $\mathrm{H}$-atom parameters, complete distances and angles, anisotropic temperature parameters and observed and calculated structure factors have been deposited with the British Library Document Supply Centre as Supplementary Publication No. SUP 55055 (22 pp.). Copies may be obtained through The Technical Editor, International Union of Crystallography, 5 Abbey Square, Chester $\mathrm{CH} 1$ 2HU, England. [CIF reference: HH0585]

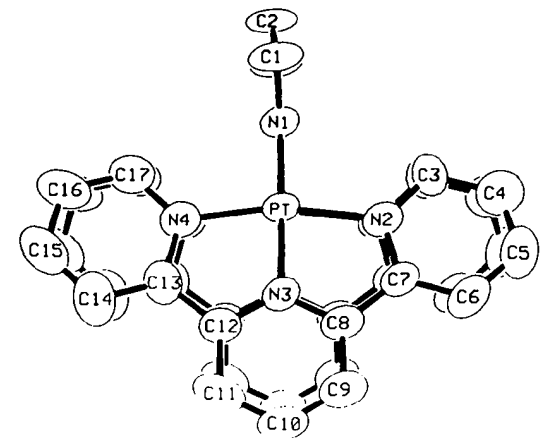

Fig. 1. A labeled ORTEPII (Johnson, 1976) drawing of the cation with $50 \%$ probability ellipsoids viewed down the intermetal axis. $\mathrm{H}$ atoms are not shown.
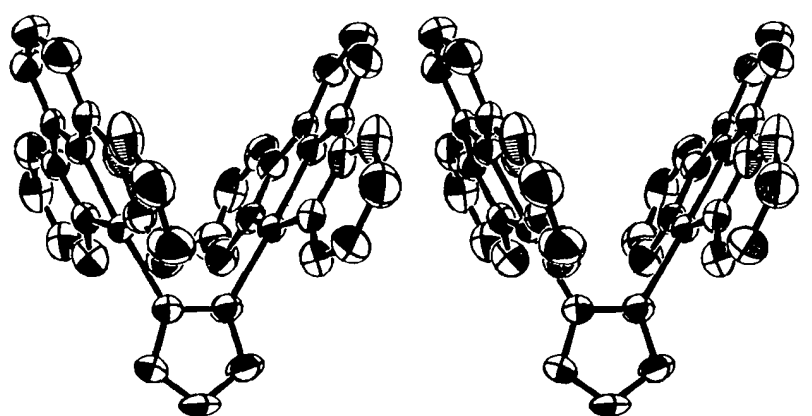

Fig. 2. An ORTEPII (Johnson, 1976) stereoscopic drawing showing the complete cation. Atoms are shown as $50 \%$ probability ellipsoids. 
terpyridine unit. This results in a short $\mathrm{Pt}-\mathrm{N} 3$ distance $[1.938(5) \AA]$ and an N2-Pt- N4 trans bond angle of only $162.2(2)^{\circ}$. The remaining bond lengths around the $\mathrm{Pt}$ atom are all over $2 \AA[2.018(6)$, 2.020 (5) and $2.031(6) \AA$ ]. This geometry is entirely consistent with other related $\mathrm{Pt}(\mathrm{tpy})$ complexes (Dewan, Lippard \& Bauer, 1980; Ratilla, Scott, Moxness \& Kostic, 1990; Wong \& Lippard, 1977; Jennette, Gill, Sadownick \& Lippard, 1976).

The two $\mathrm{Pt}$ atoms are bridged by a single pyrazolyl group, resulting in a Pt ‥Pt separation of 3.432 (1) $\AA$ (parallel, by symmetry, with the $y$ axis) and an inclination between the two square planes of $47.7(12)^{\circ}$. This distance is intermediate in the range of $\mathrm{Pt}^{\mathrm{II}}$... $\mathrm{Pt}^{\mathrm{II}}$ separations exhibited by other binuclear tpy complexes, that have been observed as short as $2.9884 \AA$ (Ratilla et al., 1990) and as long as $4.420 \AA$ (Dewan et al., 1980). The Pt atom is displaced out of the terpyridine plane by $c a 0.2 \AA$ towards the other $\mathrm{Pt}$ atom in the same binuclear unit. The bond lengths and angles within each pyridine ring are characteristic of a delocalized aromatic system, ranging from 1.334 (10) to 1.395 (14) $\AA$ and from 117.2 (8) to $124.9(7)^{\circ}$. Similarly, the bond lengths and angles within the pyrazolyl ring range from $1.331(13)$ to 1.340 (9) $\AA$ and from 105.1 (9) to $110.5(8)^{\circ}$, thereby demonstrating the delocalized nature of the $\pi$ electrons in the five-membered ring.

We thank Larry Henling for his assistance. This work was supported by an NSERC (Canada) Postdoctoral Fellowship to JAB and a grant from the Office of Naval Research (N00014-89-J-3198).

\section{References}

Cromer, D. T. (1974). International Tables for X-ray Crystallography, Vol. IV, pp. 149-151. Birmingham: Kynoch Press. (Present distributor Kluwer Academic Publishers, Dordrecht.)

CROMER, D. T. \& WABER, J. T. (1974). International Tables for $X$-ray Crystallography, Vol. IV, pp. 99-101. Birmingham: Kynoch Press. (Present distributor Kluwer Academic Publishers, Dordrecht.)

Dewan, J. C., Lippard, S. J. \& Bauer, W. R. (1980). J. Am. Chem. Soc. 102, 858-860.

Duchamp, D. J. (1964). CRYM Crystallographic computing system. Am. Crystallogr. Assoc. Meet., Bozeman, Montana. Paper B14, p.29.

JenNette, K. W., Gill, J. T., SADownick, J. A. \& LipPaRd, S. J. (1976). J. Am. Chem. Soc. 98, 6159-6168.

JoHnSON, C. K. (1976). ORTEPII. Report ORNL-3794, third revision. Oak Ridge National Laboratory, Tennessee, USA.

Ratilla, E. M. A., Scott, B. K., Moxness, M. S. \& Kostic, N. M. (1990). Inorg. Chem. 29, 918-926.

Wong, Y.-S. \& LiPPaRd, S. J. (1977). J. Chem. Soc. Chem. Commun. pp. 824-825.

Acta Cryst. (1992). C48, 1422-1425

\title{
Structure of (Benzenethiolato)oxo[ $N$-(2-sulfidophenyl)salicylidene- iminato(2-)-O,N,S|technetium $(\mathrm{V})^{*}$
}

\author{
By G. BANDOLI AND U. MAZzI \\ Dipartimento di Scienze Farmaceutiche, Università di Padova, Via Marzolo 5, 35131 Padova, Italy \\ AND H.-J. PIETZSCH AND H. SPIES $\dagger$ \\ Central Institute of Nuclear Research, Rossendorf, D-8051 Dresden, POB 19, Germany
}

(Received 19 September 1991; accepted 12 December 1991)

\begin{abstract}
Tc}\left(\mathrm{C}_{13} \mathrm{H}_{9} \mathrm{NOS}\right)(\mathrm{O})\left(\mathrm{C}_{6} \mathrm{H}_{5} \mathrm{~S}\right)\right], \mathrm{C}_{19} \mathrm{H}_{14} \mathrm{NO}_{2}$ $\mathrm{S}_{2} \mathrm{Tc}, M_{r}=451.2$, monoclinic, $P 2_{1}, a=7.901$ (1), $b=$ $10.147(1), \quad c=11.370(2) \AA, \quad \beta=93.60(1)^{\circ}, \quad V=$ $909.8(2) \AA^{3}, Z=2, D_{x}=1.646 \mathrm{~g} \mathrm{~cm}^{-3}, \lambda(\mathrm{Mo} K \alpha)=$ $0.71073 \AA, \mu=1 \mathrm{~mm}^{-1}, F(000)=452, T=293 \mathrm{~K}, R$ $=0.055$ for 1703 unique reflections. The tridentate dianionic ligand spans three positions in the basal plane, including the phenolic $\mathrm{O}$ atom, the neutral $\mathrm{N}$

\footnotetext{
* Systematic name: $\overline{\text { (benzenethiolato)oxo }}$ 2-[(2-sulfidophenyl)iminomethyl]phenolato- $O, N, S\}$ technetium $(\mathrm{V})$.

$\dagger$ To whom all correspondence should be addressed.
}

0108-2701/92/081422-04\$06.00 atom and the thiolate $S$ atom, the fourth position being occupied by the benzenethiolate $S$ atom. The Tc atom lies $0.66 \AA$ above the basal plane towards the $O(1)$ apex and with respect to the mean basal plane the four donor atoms are in a slightly puckered $( \pm 0.12 \AA)$ arrangement.

Introduction. Neutral Tc complexes $\mathrm{TcO}(\mathrm{S} X \mathrm{~S})(\mathrm{S} R)$, with ${ }^{-} \mathrm{S} X \mathrm{~S}^{-}=$dianionic tridentate ligands ${ }^{-} \mathrm{S}-\mathrm{CH}_{2} \mathrm{CH}_{2}-X-\mathrm{CH}_{2} \mathrm{CH}_{2}-\mathrm{S}^{-} \quad(X=\mathrm{O}, \mathrm{S})$ and $\mathrm{RS}^{-}=$monodentate thiolates were obtained by joint action of both the tridentate and the monodentate

(C) 1992 International Union of Crystallography 